\title{
ON THE DETERMINATION OF ZIGLIN MONODROMY GROUPS
}

By

\author{
Richard C. Churchill \\ and
}

David L. Rod

IMA Preprint Series \# 646

May 1990 


\section{ON THE DETERMINATION OF ZIGLIN MONODROMY GROUPS}

by

Richard C. Churchill ${ }^{1}$

Department of Mathematics

Hunter College

695 Park Avenue

New York, New York 10021

and

David L. Rod ${ }^{2}$

Department of Mathematics and Statistics

University of Calgary

Calgary, Alberta T2N 1N4

Canada

1. Author's research supported in part by NSF grant \#DMS 8802911.

2. Author's research supported in part by Natural Sciences and Engineering Research Council of Canada. Grant \#OGP0008507.

$\underline{\text { Introduction }}$

Consider a linear differential equation $y^{\prime \prime}+p(x) y^{\prime}+q(x) y=0,,^{\prime}=(d / d x)$, with rational coefficients on the Riemann sphere $\mathbb{P}^{1}$ or, more generally, a holomorphic (flat) connection on a rank two complex vector bundle over a Riemann surface (see [1]). The monodromy group can be viewed as an automorphism group of some distinguished fiber, and is called Ziglin if it preserves a non-constant rational function on this vector space (see [20]). The determination of which monodromy groups, are Ziglin is crucial in integrability questions for complex analytic Hamiltonian systems. Here we solve the problem completely for the Fuchsian case on $\mathbb{P}^{1}$, and in more general bundle contexts where symmetries allow reduction to thr first case. The key elements are a classification of Ziglin subgroups of $\mathrm{GL}(2, \mathbb{C})$ and an algorithm of J. Kovacic [10] which determines the nature of the differential Galois group of a second order equation (as above) on $\mathbb{P}^{1}$. 
The necessary background in differential Galois theory appears in Section 2, and its application to determining the Ziglin subgroups of $\mathrm{GL}(2, \mathbb{C})$ in Section 3 . The Kovacic algorithm is outlined for the Fuchsian case in Section 4, with applications to Hamiltonian systems in Section 5. For background in these group theoretical properties of differential equations we refer to $[7,9,11]$, and especially the surveys $[16,17]$.

The authors wish to thank Michael Singer for lengthy discussions on differential Galois theory, for suggesting that such methods would prove useful in Ziglin analysis, and for communicating a preliminary result which is now subsumed by Theorem 3.5(e). We would also like to thank Alberto Baider for extensive discussions on the Ziglin group classification problem, and for communicating Corollary 3.4 which motivated Theorem 3.3. Comments on the second example of Section 5 by Haruo Yoshida are also gratefully acknowledged. Finally, we would like to thank the Director and staff of the Institute for Mathematics and Its Applications at the University of Minnesota, Minneapolis, for their hospitality while this work was carried out. After this paper was completed the authors became aware of the recent thesis of Morales [21] which also considers Ziglin analysis in terms of differential Galois theory, but with a different emphasis, and without Kovacic's algorithm. We refer the reader to this work for its many nice examples and as complementary reading.

\section{Motivation}

Let $\mathrm{X}$ be a Riemann surface and $\nabla$ a connection on a rank two complex vector bundle $\pi_{\mathrm{E}}: \mathrm{E} \rightarrow \mathrm{X}$. To each loop $\gamma$ in $\mathrm{X}$ based at $\mathrm{x}_{0} \varepsilon \mathrm{X}$ assign the automorphism of the fiber $\mathrm{E}_{0}=\pi_{\mathrm{E}}^{-1}\left(\left\{\mathrm{x}_{0}\right\}\right)$ which results on sending $\mathrm{v} \varepsilon \mathrm{E}_{0}$ to the endpoint of the horizontal lift of $\gamma^{-1}$ issuing from v. Since $\nabla$ is flat this lift depends only on the homotopy class of $\gamma$ in $\pi_{1}\left(\mathrm{X}, \mathrm{x}_{0}\right)$, and defines the monodromy representation $\rho$ : $\pi_{1}\left(\mathrm{X}, \mathrm{x}_{0}\right) \rightarrow \operatorname{Aut}\left(\mathrm{E}_{0}\right)$. The image $\mathrm{G}_{\mathrm{M}}=\rho\left(\pi_{1}\left(\mathrm{X}, \mathrm{x}_{0}\right)\right)$ is the monodromy group of $\nabla$ at $x_{0}$. Different choices of basepoint give isomorphic monodromy groups, and given a basis of $E_{0}$ one can identify $G_{M}$ with a subgroup of $G L(2, \mathbb{C})$. In the classical case $X$ $=\mathbb{C} \backslash\{$ finite set $\}$ and $\nabla$ can be viewed as a linear ordinary differential equation on the 
complex plane with possible singularities on this finite set as well as at $\infty$ (see $[4, \mathrm{p}$. 93]). Then $\mathrm{E}_{0}$ is identified with the initial values (and hence germs at $x_{0}$ ) of solutions, and $\rho$, and hence $\mathrm{G}_{M}$, are defined by analytic continuation of these solutions along $\gamma^{-1}$.

Throughout this paper any function said to be an "integral" will be assumed non-constant. Thus $\mathrm{G}_{\mathrm{M}}$ has an integral $\mathrm{f}$, or is a Ziglin group, if there is a non-constant rational function $f$ on $E_{0}$ which is preserved by the action; that is, $g^{*} f$ $=f$ for all $g \in G_{M}$. An equivalent condition is the existence of an integral $F$ for $\nabla$; that is, a meromorphic function $F$ on $E$ which is rational on fibers and constant along horizontal lifts of curves in $\mathrm{X}$. Indeed, $f=F \mid E_{0}$ will be preserved by $G_{M}$, hence $G_{M}$ will be Ziglin, and any such $f$ can be uniquely extended to a corresponding integral $\mathrm{F}$ of $\nabla$ through "parallel transport".

\subsection{Example (Ziglin theory for two degree-of-freedom Hamiltonian systems):}

Let $\mathrm{M}$ be a complex symplectic 4-manifold and $\Gamma$ a non-equilibrium phase curve, within an energy surface $\Sigma$, of a holomorphic Hamiltonian vector field $\mathrm{X}_{H}$ on $M$. Linearization along $\Gamma$ induces a holomorphic (hence flat) connection $\nabla$ on the normal bundle $\mathrm{N}=(\mathrm{T} \Sigma \mid \Gamma) / \mathrm{T} \Gamma$ of $\Gamma$ in $\Sigma$, called the normal variational equation (NVE). By a result of Ziglin [20] any meromorphic integral of $\mathrm{X}_{\mathrm{H}}$ independent on a neighborhood (in $M$ ) of $\Gamma$ (but not necessarily on $\Gamma$ itself) will induce an integral for $\nabla$; hence $G_{M}$ will be a Ziglin group. Therefore, if $G_{M}$ is not a Ziglin group, $X_{H}$ cannot be integrable.

When reasonable symmetries are present a connection can be reduced. Specifically, suppose a finite $\mathrm{i} \cup \mathrm{u} p \mathrm{G}$ acts $\pi_{\mathrm{E}}$-equivariantly on $\mathrm{E} \rightarrow \mathrm{X}$, freely and properly discontinuously on $\mathrm{X}$, and linearly on fibers. Then $\mathrm{X} / \mathrm{G}$ is again a Riemann surface and the connection $\tilde{\nabla}=(1 /|\mathrm{G}|) \sum_{\mathrm{g} \in \mathrm{G}} \mathrm{g}^{*} \nabla$ is preserved by $\mathrm{G}$, thus inducing a connection $\nabla_{G}$ on $E / G \rightarrow X / G$. We say $\nabla$ is symmetric (w.r.t. G) if $\nabla=\tilde{\nabla}$. 
1.2 Theorem: Let $\nabla$ be symmetric w.r.t. the finite group $G$ which acts on $\mathrm{E} \rightarrow \mathrm{X}$ as above. Then $\nabla$ admits an integral iff this is the case for $\nabla_{G}$. In particular, the monodromy group of $\nabla$ is Ziglin iff the monodromy group of $\nabla_{G}$ is Ziglin.

Proof: (a) Assume $\nabla$ admits an integral. It suffices to show that the existence of an integral $F$ for $\nabla$ implies the existence of a G-invariant integral. We do this by adapting an argument from [20, p. 186] which deals with a related situation.

Let $G=\left\{g_{j}\right\}_{j=1}^{m}$ with $g_{1}=i d$, and set $f_{j}=g_{j}^{*}$ F. Assume $\left\{f_{1}, \ldots, f_{k}\right\}$ are the distinct $f_{j}$, and let $m_{j}$ be the number of occurrences of $f_{j}$ in $\left\{f_{1}, \ldots, f_{m}\right\}$. Now consider the G-invariant integrals $\psi_{i}=\underset{j=1}{m}\left(f_{j}\right)^{i}$ of $\nabla$. Since

$$
\operatorname{det} \frac{\partial\left(\psi_{1}, \ldots, \psi_{k}\right)}{\partial\left(f_{1}, \ldots, f_{k}\right)}=(k !)\left[\prod_{j=1}^{k} m_{j}\right] \prod_{1 \leq i<j \leq k}\left(f_{j}-f_{i}\right) \neq 0
$$

(the last term is a standard Vandermonde determinant), we can solve locally for the $f_{i}$ as analytic functions of the $\left\{\psi_{j}\right\}$. In particular, we can write $F=f_{1}$ locally as an analytic function of the $\left\{\psi_{\mathrm{j}}\right\}$, and when restricted to a fiber we then have $0 \neq \mathrm{dF}=$ $\sum \mathrm{a}_{\mathrm{j}} \mathrm{d} \psi_{\mathrm{j}}$ for appropriate $\mathrm{a}_{\mathrm{j}}$. We conclude that some $\psi_{\mathrm{j}}$ must be non-constant and hence a symmetric integral for $\nabla$.

(b) The converse of (a) follows from the fact that the monodromy group of $\nabla$ can be identified with a subgroup of the monodromy group of $\nabla_{G}$ (see [4, Proposition 1.1, p. 94]) and the comments preceding Example 1.1 on the relation between integrals of the monodromy group and integrals of the connection.

Q.E.D.

$1.3 \mathrm{Cc}$ videy: Assume the connection $\nabla$ on $\mathrm{N} \rightarrow \Gamma$ of Example 1.1 is symmetric w.r.t. a finite group $\mathrm{G}$ acting as in Theorem 1.2. If the monodromy group of the reduced connection $\nabla_{G}$ is not Ziglin, then $X_{H}$ has no meromorphic integral independent of $\mathrm{H}$. 
We will see an example of Corollary 1.3 in Section 5. We refer to [3] and [4] for examples of symmetric connections. In particular, [3, Section 4] presents a general theory concerning when a given connection is symmetric. In examples the group $\mathrm{G}$ is often given as an action on the base space $\mathrm{X}$; Proposition 2.3 in [4] shows when this can be lifted to a $\pi_{\mathrm{E}}$-equivariant action on $\mathrm{E} \rightarrow \mathrm{X}$ under which $\nabla$ is symmetric (see also Section 4 of [3]).

In the next few sections we will assume that reduction has transformed $\nabla$ to a connection $\nabla_{G}$ which can be viewed as a linear ordinary differential equation on $\mathbb{P}^{1}$. When $\nabla_{G}$ is Fuchsian we show that the monodromy group of $\nabla_{G}$ is Ziglin iff the differential Galois group of $\nabla_{G}$ is Ziglin. We then adapt an algorithm of Kovacic [10] (see also [5]) in Section 4 to determine if the differential Galois group has this property.

\section{Preliminaries on Differential Galois Theory}

In this section we collect some standard results on differential Galois theory and algebraic subgroups of $\mathrm{SL}(2, \mathbb{C})$ needed in later sections. Let $\mathbb{C}(\mathrm{x})$ denote the field of rational functions in $\mathrm{x}$ with coefficients in $\mathbb{C}$ and consider the linear ordinary differential equation.

$$
\mathrm{y}^{\prime \prime}=\mathrm{r}(\mathrm{x}) \mathrm{y}, \quad{ }^{\prime}=(\mathrm{d} / \mathrm{dx})
$$

on the Riemann sphere $\mathbb{P}^{1}$, where $r(x) \varepsilon \mathbb{C}(x)$. Let $\Lambda$ denote the set of poles of $r(x)$, set $X=\mathbb{P}^{1} \backslash(\wedge \cup\{\infty\})$, and fix $x_{0} \varepsilon X$.

$\mathscr{M}$ will denote the field of germs of meromorphic functions at $\mathrm{x}_{0}$, which we view as an extension of $\mathbb{C}(\mathrm{x})$ by identifying the latter with the germs of such functions at $\mathrm{x}_{0} . \mathrm{V} \subset \mathcal{K}$ will denote the linear space of germs of solutions of (2.1) at $\mathrm{x}_{0}, \mathrm{~V}^{\prime} \subset \mathcal{H}$ the associated derivatives, and $\mathrm{E} \subset \mathcal{H}$ the extension of $\mathbb{C}(\mathrm{x})$ generated by $\mathrm{V} \cup \mathrm{V}^{\prime}$. $\mathrm{E}$ is the Picard-Vessiot extension of $\mathbb{C}(\mathrm{x})$ associated to $(2.1)$, and the differential Galois group $\mathrm{G}_{\mathrm{D}}=\mathrm{G}(\mathrm{E} / \mathbb{C}(\mathrm{x}))$ of that equation is the group of 
automorphisms of $\mathrm{E}$ which fix $\mathbb{C}(\mathrm{x})$ and commute with differentiation. Elements of $G_{D}$ are determined by their action on $V$, hence $G_{D}$ may be viewed as a subgroup of Aut(V).

A subgroup of $\operatorname{Aut}(\mathrm{V}) \simeq \mathrm{GL}(2, \mathbb{C})$ is (1) reducible (or triangulizable) if it is conjugate to a (lower) triangular subgroup of $\mathrm{GL}(2, \mathbb{C}) ;(2)$ a $D P$-group if it is conjugate to a subgroup of $\left\{\left(\begin{array}{ll}\alpha & 0 \\ 0 & \beta\end{array}\right)\right\} \cup\left\{\left(\begin{array}{ll}0 & \gamma \\ \delta & 0\end{array}\right)\right\} \subset \mathrm{GL}(2, \mathbb{C})$. (The latter terminology for "diagonal-permutation" is from [2].) Note that a diagonalizable group is both reducible and a DP-group.

2.2 Proposition: Let $G_{N}$ be the monodromy group of (2.1) and $G_{D}$ its differential Galois group. Then:

(a) $G_{D}$ is an algebraic unimodular subgroup of $A u t(V)$. In particular, it is Zariski closed in this space.

(b) Any element of $E$ fixed by all elements of $G_{D}$ must be in $\mathbb{C}(x)$.

(c) $G_{N} \subset G_{D}$.

Moreover, if (2.1) is Fuchsian we have:

(d) $\bar{G}_{N}=G_{D}$, i.e., $G_{N}$ is Zariski dense in $G_{D}$;

(e) $G_{N}$ is reducible, diagonalizable, or a DP-group iff $G_{D}$ is such; and

(f) $G_{N}$ is finite iff $G_{D}$ is such, in which case $G_{N}=G_{D}$.

Proof: (a) and (b) are standard (e.g., see [9, pp. 36, 38, and 41]). 
(c) (Adapted from [18].) Analytic continuation along the inverse of any loop in $\mathrm{X}$ based at $\mathrm{x}_{0}$ defines an element of $G_{\mathrm{N}}$ and one of $G_{D}$, and these are identical when viewed in $\operatorname{Aut}(\mathrm{V})$.

(d) This is Proposition III of [18].

(e) and (f) are immediate from (c) and (d).

Q.E.D.

2.3 Proposition: An algebraic subgroup of $\mathrm{SL}(2, \mathbb{C})$ is either

Case I: reducible;

Case II: a DP-group;

Case III: a finite group which, if not of Case I or II, must be projectively (i.e., mod tid) the tetrahedral, octahedral, or icosahedral group; or

Case IV: $\quad \mathrm{SL}(2, \mathbb{C})$.

In particular, the differential Galois group $G_{D}$ of $(2.1)$ must have one of these forms.

Proof:
See $[10$, pp. 7 and 27$] . \quad$ Q.E.D.

2.4 Proposition: If every element of an algebraic subgroup of $\mathrm{GL}(\mathrm{n}, \mathbb{C})$ has finite order, then that group must be finite.

Proof: $\quad$ This is a lemma on p. 1328 of [18]. Q.E.D.

3. Ziglin Subgroups of $\mathrm{GL}(2, \mathbb{C})$

Application of Corollary 1.3 when the group $G$ is not symplectic may yield a 
reduced connection $\nabla_{G}$ which is Fuchsian on $\mathbb{P}^{1}$ but not of the form (2.1). The monodromy of $\nabla_{G}$ will then be a subgroup of $\mathrm{GL}(2, \mathbb{C})$ rather than $\mathrm{SL}(2, \mathbb{C})$. The purpose of this section is to develop relationships (see Theorem 3.5 and Corollary 3.6 below) which allow us to exploit the classification of algebraic subgroups of $\operatorname{SL}(2, \mathbb{C})$ given in Proposition 2.3 (which should be compared with Corollary 3.4 below).

Recall from Section 1 that a subgroup Z C GL(2,C) is Ziglin, or a Ziglin group, if there is a non-constant rational function $f: \mathbb{C}^{2} \rightarrow \mathbb{C}$ preserved by $Z$; i.e., such that $\mathrm{g}^{*} \mathrm{f}=\mathrm{f}$ for all $\mathrm{g} \varepsilon \mathrm{Z}$.

\subsection{Proposition:}

(a) Any conjugate of a Ziglin group $\mathrm{Z}$ is again Ziglin.

(b) Any subgroup of a Ziglin group is again Ziglin.

(c) The Zariski closure $\bar{Z}$ of any Ziglin subgroup $\mathrm{Z}$ preserves any rational function $\mathrm{f}$ preserved by $\mathrm{Z}$. In particular, $\overline{\mathrm{Z}}$ must be Ziglin.

(d) A subgroup $\mathrm{Z} \subset \mathrm{GL}(2, \mathbb{C})$ is Ziglin iff the Zariski closure $\overline{\mathrm{Z}}$ is Ziglin.

Proof: $\quad$ (a) $\mathrm{g}^{* \mathrm{f}}=\mathrm{f}$ implies $\left(\mathrm{h}^{-1} \mathrm{gh}\right)^{*} \mathrm{~h}^{*} \mathrm{f}=\mathrm{h}^{*} \mathrm{f}$ for any $\mathrm{h} \varepsilon \mathrm{GL}(2, \mathbb{C})$.

(b) Obvious.

(c) Write $\mathrm{f}=(\mathrm{p} / \mathrm{q})$ where $\mathrm{p}$ and $\mathrm{q}$ are polynomials and fix $\mathrm{w} \in \mathbb{C}^{2}$. Then $\mathrm{P}_{\mathrm{w}}(\mathrm{g})=\left(\mathrm{g}^{*} \mathrm{p}\right)(\mathrm{w}) \cdot \mathrm{q}(\mathrm{w})-\mathrm{p}(\mathrm{w}) \cdot\left(\mathrm{g}^{*} \mathrm{q}\right)(\mathrm{w})$ is a polynomial vanishing on $\mathrm{Z}$, hence $\bar{Z}$ is inntained in the zero set of $P_{w}$. Since w was arbitrary the result follows.

(d) By (b) and (c).

Q.E.D. 
Now let $\mathrm{Z} \subset \mathrm{GL}(2, \mathbb{C})$ be Ziglin and let $\mathrm{f}=(\mathrm{p} / \mathrm{q})$ be a non-constant rational function preserved by $\mathrm{Z}$. Then we can assume

$$
f(x, y)=\underset{j=1}{\stackrel{r}{\prod}}\left(x-\lambda_{j} y\right)^{m_{j}}
$$

where the $\left\{\lambda_{j}\right\}$ are distinct and $\mathrm{m}_{\mathrm{j}} \in \mathbb{Z} \backslash\{0\}$. Indeed, $\mathrm{g}^{*} \mathrm{f}=\mathrm{f}$ for $\mathrm{g} \varepsilon \mathrm{Z}$ iff $\left(\mathrm{g}^{*} \mathrm{p}\right) \cdot \mathrm{q}$ $=\mathrm{p} \cdot\left(\mathrm{g}^{*} \mathrm{q}\right)$, and by comparing lowest (or highest) order terms in this last expression we see that $\mathrm{p}$ and $\mathrm{q}$ may be assumed homogeneous. The expression (3.2) then follows by factoring and, if necessary, conjugating by $\mathrm{h}=\left(\begin{array}{ll}0 & 1 \\ 1 & 0\end{array}\right)$. Note that such a factorization does not generally hold for homogeneous polynomials in three or more variables (e.g., see [6, pp. 50-51]).

3.3 Theorem: The Ziglin subgroups of $\mathrm{GL}(2, \mathbb{C})$ are precisely those that can be conjugated to a subgroup of one of the following three groups:

(1) $\mathrm{T}(\mathrm{n})=\left\{\left[\begin{array}{ll}\alpha & 0 \\ \delta & \beta\end{array}\right] \mid \alpha^{\mathrm{n}}=1\right\}$,

(2) $\mathrm{T}(\mathrm{m}, \mathrm{n})=\left\{\left[\begin{array}{ll}\alpha & 0 \\ 0 & \beta\end{array}\right] \mid \alpha^{\mathrm{m}} \beta^{\mathrm{n}}=1\right\}$,

(3) $\mathrm{D}(\mathrm{n})=\left\{\left[\begin{array}{ll}\alpha & 0 \\ 0 & \beta\end{array}\right] \mid(\alpha \beta)^{\mathrm{n}}=1\right\} \cup\left\{\left[\begin{array}{ll}0 & \gamma \\ \delta & 0\end{array}\right] \mid(\delta \gamma)^{\mathrm{n}}=1\right\}$,

and

(4) the projectively finite groups.

Proof: Let $\mathrm{Z}$ be a Ziglin subgroup of $\mathrm{GL}(2, \mathbb{C})$ that fixes the rational function (3.2). We havie three cases.

Case I $(r=1)$ : $\quad z$ then fixes the line $x=\lambda_{1} y$, hence there is a conjugacy $h$ so that $\left(h^{*} f\right)(x, y)=x^{n}$ (on setting $m_{1}=n$ ) and $\left(h^{-1} Z h\right) \subset T(n)$. Conversely, $\left(h^{-1} Z_{0} h\right)$ C $\mathrm{T}(\mathrm{n})$ implies the subgroup $\mathrm{Z}_{0}$ preserves $\left(\mathrm{h}^{-1}\right)^{*}\left(\mathrm{x}^{\mathrm{n}}\right)$. 
Case II $(r=2)$ : $\quad$ Each $g \in Z$ will preserve or permute the two lines $x=\lambda_{j} y, j$ $=1,2$. There is then a conjugacy $\mathrm{h}$ so that $\left(\mathrm{h}^{*} \mathrm{f}\right)(\mathrm{x}, \mathrm{y})=\mathrm{x}^{\mathrm{m}} \mathrm{y}^{\mathrm{n}}$ (on setting $\mathrm{m}_{1}=\mathrm{m}$, $\left.m_{2}=n\right)$ and $\left(h^{-1} Z h\right) c\left\{\left[\begin{array}{ll}\alpha & 0 \\ 0 & \beta\end{array}\right]\right\} \cup\left\{\left[\begin{array}{ll}0 & \gamma \\ \delta & 0\end{array}\right]\right\}$. If there are no elements of the form $\mathrm{g}=\left[\begin{array}{ll}0 & \gamma \\ \delta & 0\end{array}\right]$ in $\left(\mathrm{h}^{-1} \mathrm{Zh}\right)$, then $\alpha^{\mathrm{m}} \beta^{\mathrm{n}}=1$ implies $\left(\mathrm{h}^{-1} \mathrm{Zh}\right) \subset \mathrm{T}(\mathrm{m}, \mathrm{n})$. If there is such an element $\mathrm{g}$ then $\left(\mathrm{g}^{*} \mathrm{f}\right)(\mathrm{x}, \mathrm{y})=\gamma^{\mathrm{m}} \delta^{\mathrm{n}} \mathrm{x}^{\mathrm{n}} \mathrm{y}^{\mathrm{m}}=\mathrm{x}^{\mathrm{m}} \mathrm{y}^{\mathrm{n}}$ implies $\mathrm{m}=\mathrm{n}$ and $\left(\mathrm{h}^{-1} \mathrm{Zh}\right) \subset \mathrm{D}(\mathrm{n})$. Conversely, if $\left(h^{-1} Z_{0} h\right)$ is a subgroup of (2) or (3) above, then $Z_{0}$ preserves $\left(h^{-1}\right)^{*}\left(x^{m} y^{n}\right)$, with $\mathrm{m}=\mathrm{n}$ for case $(3)$.

Case III $(\mathrm{r} \geq 3)$ : $\quad$ Let $\mathrm{K}=\{\mathrm{c} \cdot \mathrm{id} \mid \mathrm{c} \quad \epsilon \quad \mathbb{C} \backslash\{0\}\}$. We must show that the projectivization $\mathrm{PZ}=\mathrm{Z} /(\mathrm{Z} \cap \mathrm{K})$ is finite. There is a positive integer $\mathrm{n}$ so that for all $g \in Z$ the element $g^{n}$ fixes each of the lines $x=\lambda_{j} y(j=1, \ldots, r)$. Since $r \geq 3$, this forces $\mathrm{g}^{\mathrm{n}}=\mathrm{c}(\mathrm{g}) \cdot \mathrm{id}$ where $\mathrm{c}(\mathrm{g})$ is a constant dependent on $\mathrm{g}$.

(a) If in (3.2) we have $\sum_{j=1}^{\mathrm{r}} \mathrm{m}_{\mathrm{j}} \neq 0$, then $\left(\mathrm{g}^{\mathrm{n}}\right)^{*} \mathrm{f}=\mathrm{f}$ implies each $\mathrm{c}(\mathrm{g})$ is a root of unity. By Proposition 3.1(c) we may replace $\mathrm{Z}$ by the algebraic group $\overline{\mathrm{Z}}$, hence $\mathrm{Z}$ itself must be finite by Proposition 2.4 .

(b) Now assume $\sum_{j_{-}=1}^{\mathrm{r}} \mathrm{m}_{\mathrm{j}}=0$ in (3.2). Then PZ preserves (3.2), and applying the last part of the argument in (a) above to the Zariski closure of $[\mathrm{PZ} U$ $(-1) \mathrm{PZ}$ ] (which we can think of as a subgroup of $\mathrm{SL}(2, \mathbb{C})$ and which also preserves $\mathrm{f}$ ), we see that $\mathrm{PZ}$ is finite.

Conversely, any subgroup $\mathrm{Z}_{0} \subset \mathrm{GL}(2, \mathbb{C})$, for which the projectivization $\mathrm{PZ}_{0}$ is finite, preserves the rational function $f_{\lambda}(x, y)$ where $f_{\lambda}=\left(\Pi g^{*} x\right) /\left(\Pi g^{*}(x-\lambda y)\right)$ and the products are taken over $\mathrm{g} \in \mathrm{PZ}_{0}$. One then chooses the parameter $\lambda \in \mathbb{C} \backslash\{0\}$ so that $f_{\lambda}$ is non-trivial (note that one has to be careful on this point since, for 
example, $\left(\Pi g^{*} x\right) /\left(\Pi g^{*} y\right)=1$ for $g$ in the 2-element group $\left.\left\{\left[\begin{array}{ll}1 & 0 \\ 0 & 1\end{array}\right],\left[\begin{array}{ll}0 & 1 \\ 1 & 0\end{array}\right]\right\}\right)$.

Q.E.D.

3.4 Corollary: The Ziglin subgroups of $\mathrm{SL}(2, \mathbb{C})$ are precisely those that can be conjugated to a subgroup of one of the following two groups:

(1) $\mathrm{T}(\mathrm{n}) \cap \mathrm{SL}(2, \mathbb{C})$,

(2) $\mathrm{D}(\mathrm{n}) \cap \mathrm{SL}(2, \mathbb{C})$,

and

(3) the finite groups.

Proof: $\quad T(m, n) \cap \operatorname{SL}(2, \mathbb{C}) \subset T(m-n) \cap S L(2, \mathbb{C})$ since $\alpha \beta=1$.

Q.E.D.

The format of the next theorem follows that in the algorithm due to Kovacic [10] which we explain in Section 4. The assumption on the $\left\{\theta_{j}\right\}$ in Proposition 3.6 is a natural one for many applications. For the definitions of $G_{N}$ and $G_{M}$ see Section 2.

$\underline{3.5}$ Theorem: For $\theta_{j} \in \mathbb{C} \backslash\{0\}$ let $N=\left\{N_{j}\right\}_{j=1}^{k} \subset \operatorname{GL}(2, \mathbb{C})$ and $M=\left\{M_{j}=\theta_{j} N_{j}\right\}_{j=1}^{k}$ generate the respective groups $G_{N}$ and $G_{M}$. Then $G_{N}$ and $G_{M}$ are simultaneously reducible, diagonalizable, or DP-groups. Moreover, they are simultaneously finite iff all $\theta_{\mathrm{j}}$ are roots of unity.

Now assume $G_{N} \subset S L(2, \mathbb{C})$. Then:

(a) If $\mathrm{G}_{\mathrm{N}}$ is reducible, but not diagonalizable, under a conjugacy $\mathrm{h}$ for which $\mathrm{h}^{-1} \mathrm{~N}_{\mathrm{j}} \mathrm{h}=\left[\begin{array}{ll}\lambda_{\mathrm{j}} & 0 \\ * & \lambda_{\mathrm{j}}^{-1}\end{array}\right]$, then $\mathrm{G}_{\mathrm{M}}$ is Ziglin iff all $\theta_{\mathrm{j}} \lambda_{\mathrm{j}}$ are roots of unity. 
(b) If $G_{N}$ is diagonalizable with $h^{-1} N_{j} h=\left[\begin{array}{cc}\lambda_{j} & 0 \\ 0 & \lambda_{j}^{-1}\end{array}\right]$, then $G_{M}$ is Ziglin iff all $\theta_{\mathrm{j}} \lambda_{\mathrm{j}}$ are roots of unity or there are integers $\mathrm{m}$ and $\mathrm{n}$ such that $\left(\theta_{\mathrm{j}}\right)^{\mathrm{m}+\mathrm{n}}$. $\left(\lambda_{j}\right)^{m-n}=1$ for all $j$.

(c) If $G_{N}$ is a DP-group, but is not reducible, with $h^{-1} N_{j} h=\left[\begin{array}{cc}\lambda_{j} & 0 \\ 0 & \lambda_{j}^{-1}\end{array}\right]$ or $\left[\begin{array}{cc}0 & \rho_{\mathrm{j}} \\ -\rho_{\mathrm{j}}^{-1} & 0\end{array}\right]$, then $\mathrm{G}_{\mathrm{M}}$ is Ziglin iff all $\theta_{\mathrm{j}}$ are roots of unity.

(d) If $G_{N}$ is finite then $G_{M}$ is Ziglin.

(e) If none of the above cases hold for $G_{N}$, then $G_{M}$ is not Ziglin.

Proof: The initial statements concerning reducibility, diagonalizability, the DP-structure, and finiteness are clear. Statements (a)-(d) follow from the statement and proof of Theorem 3.3 (in (c) one must use the presence of an element $\left.\left[\begin{array}{cc}0 & \rho_{\mathrm{j}} \\ -\rho_{\mathrm{j}}^{-1} & 0\end{array}\right]\right)$

(e) Assume $G_{M}$ is Ziglin. Then the assumptions on $G_{N}$ imply by Proposition 2.3 that the Zariski closure $\overline{\mathrm{G}}_{\mathrm{N}}=\mathrm{SL}(2, \mathbb{C})$ and by Theorem 3.3 that the projectivization $P G_{M}$ is finite. One can then readily construct a homogeneous polynomial that vanishes on $P G_{M}$ and hence on $G_{M}$ and $G_{N}$, but not on $\operatorname{SL}(2, \mathbb{C})$, contradicting the fact that $\overline{\mathrm{G}}_{\mathrm{N}}=\mathrm{SL}(2, \mathbb{C})$.

Q.E.D.

3.6 Proposition: If all $\left\{\theta_{\mathrm{j}}\right\}_{\mathrm{j}=1}^{\mathrm{k}}$ are roots of unity, then $G_{\mathrm{N}}$ is Ziglin iff $G_{M}$ is Ziglin. 
Proof: There is an integer $d$ so that $\left(\theta_{\mathrm{j}}\right)^{\mathrm{d}}=1$ for all $\mathrm{j}$. Now raise the respective polynomials in the proof of Theorem 3.3 to the $d$-th power.

Q.E.D.

\section{The Algorithm}

Here we show how to determine if the monodromy group $G_{M} \subset \operatorname{GL}(2, \mathbb{C})$ of a second order Fuchsian equation

$$
\mathrm{z}^{\prime \prime}+\mathrm{p}(\mathrm{x}) \mathrm{z}^{\prime}+\mathrm{q}(\mathrm{x}) \mathrm{z}=0, \quad{ }^{\prime}=(\mathrm{d} / \mathrm{dx})
$$

on $\mathbb{P}^{1}$ is a Ziglin group. This is done with the aid of an algorithm due to Kovacic [10] which decides which of the cases (a) - (e) of Theorem 3.5 holds for (4.1) in its normal form

$$
y^{\prime \prime}=r(x) y, \quad r(x)=-\left[q(x)-(1 / 4) p^{2}(x)-(1 / 2) p^{\prime}(x)\right], \quad=(d / d x)
$$

which we note is also Fuchsian. We need to establish some notation.

In (4.1) we have

$$
p(x)=\sum_{j=1}^{k} \frac{A_{j}}{\left(x-a_{j}\right)}, q(x)=\sum_{j=1}^{k} \frac{B_{j}}{\left(x-a_{j}\right)^{2}}+\sum_{j=1}^{k} \frac{C_{j}}{\left(x-a_{j}\right)}, \quad \sum_{j=1}^{k} C_{j}=0 .
$$

This implies that in (4.2) we have

$$
r(x)=\sum_{j=1}^{k} \frac{\beta_{j}}{\left(x-a_{j}\right)^{2}}+\sum_{j=1}^{k} \frac{\delta_{j}}{\left(x-a_{j}\right)}, \quad \sum_{j=1}^{k} \delta_{j}=0,
$$

where, on setting $A_{\infty}=\sum_{j=1}^{k} A_{j}, \quad B_{\infty}=\sum_{j=1}^{k}\left(B_{j}+C_{j} a_{j}\right)$, and $\beta_{w}=\sum_{j=1}^{k}\left(\beta_{j}+\delta_{j} a_{j}\right)$, 


$$
\left\{\begin{array}{l}
\beta_{j}=(1 / 4)\left[\left(1-A_{j}\right)^{2}-4 B_{j}-1\right], \\
\delta_{j}=-C_{j}+(1 / 2) A_{j}\left[\sum_{i \neq j}^{k} \frac{A_{i}}{\left(a_{j}-a_{i}\right)}\right], \\
\beta_{\infty}=(1 / 4)\left[\left(1-A_{\infty}\right)^{2}-4 B_{\infty}-1\right] .
\end{array}\right.
$$

Note that the characteristic exponents of (4.2) are

$$
\left\{\begin{aligned}
\tau_{j}^{ \pm} & =(1 / 2)\left[1 \pm\left\{1+4 \beta_{j}\right\}^{\frac{1}{2}}\right]=(1 / 2)\left[1 \pm\left\{\left(1-A_{j}\right)^{2}-4 B_{j}\right\}^{\frac{1}{2}}\right] \text { at } a_{j} \\
\tau_{\infty}^{ \pm} & =(1 / 2)\left[1 \pm\left\{1+4 \beta_{\infty}\right\}^{\frac{1}{2}}\right]=(1 / 2)\left[1 \pm\left\{\left(1-A_{\infty}\right)^{2}-4 B_{\infty}\right\}^{\frac{1}{2}}\right] \text { at } \infty
\end{aligned}\right.
$$

Let $\Lambda=\left\{\mathrm{a}_{1}, \ldots, \mathrm{a}_{\mathrm{k}}\right\}$ be the finite poles of $\mathrm{p}(\mathrm{x})$ and $\mathrm{q}(\mathrm{x})$ as in (4.3), set $\mathrm{X}=$ $\mathbb{P}^{\wedge} \backslash(\wedge \cup\{\infty\})$, and fix $x_{0} \varepsilon X$. For each point $a_{j} \varepsilon \wedge$ let $\gamma_{j}$ be a positively oriented loop in $\mathrm{X}$ based at $\mathrm{x}_{0}$ that encircles only $\mathrm{a}_{\mathrm{j}}$; then $\pi_{1}\left(\mathrm{X}, \mathrm{x}_{0}\right)$ is freely generated by $\left\{\gamma_{j}\right\}_{j=1}^{k}$. Also, let $\gamma_{\infty}$ be a corresponding loop around o satisfying $\left[\begin{array}{c}k \\ \Gamma_{i=1}\end{array} \gamma_{j}\right] \gamma_{\infty}=I$. Then the monodromy representation $\rho_{\mathrm{M}}: \pi_{1}\left(\mathrm{X}, \mathrm{x}_{0}\right) \rightarrow \mathrm{GL}(2, \mathbb{C})$ of (4.1) with $\mathrm{M}_{\mathrm{j}}=$ $\rho_{M}\left(\gamma_{j}\right), M_{\infty}=\rho_{M}\left(\gamma_{\infty}\right)$, satisfies $\left[\prod_{j=1}^{k} M_{j}\right] M_{\infty}=I$, and $\left\{M_{j}\right\}_{j=1}^{k}$ generates the monodromy group $G_{M}$ of (4.1).

The poles of $\mathrm{r}(\mathrm{x})$ in (4.4) are a subset of $\wedge \cup\{\omega\}$. The monodromy representation $\rho_{\mathrm{N}}$ of $(4.2)$ has range $G_{\mathrm{N}} \subset \mathrm{SL}(2, \mathbb{C})$ and is generated by $\left\{\mathrm{N}_{\mathrm{j}}=\right.$ $\left.\rho_{\mathrm{N}}\left(\gamma_{\mathrm{j}}\right)\right\}_{\mathrm{j}=1}^{\mathrm{k}}$. Moreover (see [3, Section 6]),

$$
M_{j}=\theta_{j} N_{j}, \quad \text { where } \theta_{j}=\exp \left[-(1 / 2) \int_{\gamma_{j}^{-1}} p\right]=\exp \left(\pi i A_{j}\right)
$$

The algorithm below consists of three successive cases. Euri case is examined in turn, and lack of success in determining a solution in all three cases will correspond to (e) in Theorem 3.5 (with the first case covering both (a) and (b) of that theorem). Throughout we let $G_{D}$ denote the differential Galois group of (4.2). 
Case I (The Reducible - Diagonalizable Case):

The algorithm is phrased in terms of the "modified" characteristic exponents:

$$
\left\{\begin{array}{l}
\alpha_{j}^{ \pm}=\tau_{j}^{ \pm} \text {if } \beta_{j} \neq 0 ; \alpha_{j}^{ \pm}=1 \text { if } \beta_{j}=0, \delta_{j} \neq 0 ; \alpha_{j}^{ \pm}=0 \text { if } \beta_{j}=0=\delta_{j} \\
\alpha_{\infty}^{ \pm}=\tau_{\infty}^{ \pm} \text {if } \beta_{\infty} \neq 0 ; \alpha_{\infty}^{+}=0 \text { and } \alpha_{\infty}^{-}=1 \text { if } \beta_{\infty}=0 .
\end{array}\right.
$$

4.9 Theorem: The following two statements are equivalent:

(a) $G_{M}, G_{N}$, and $G_{D}$ are simultaneously reducible.

(b) There is a solution of (4.2) of the form $\mathrm{y}=\exp \left(\int \theta\right)$, with $\theta \in \mathbb{C}(\mathrm{x})$, which is necessarily a common eigenvector for $G_{N}$.

Moreover, there is a solution as in (b) iff

(1) there is a choice $s(j)$ and $s(\infty)$ of a plus or minus sign so that

$$
d=\left[\alpha_{\infty}^{s(\infty)}-\sum_{j=1}^{k} \alpha_{j}^{s(j)}\right] \text { is a non-negative integer; }
$$

(2) there is a unique monic, degree $d$ polynomial $P$ (which can be found by the method of_undetermined coefficients) satisfying

$$
\mathrm{P}^{\prime \prime}+2 \omega \mathrm{P}^{\prime}+\left(\omega^{\prime}+\omega^{2}-\mathrm{r}\right) \mathrm{P}=0, \text { where } \omega=\omega(\mathrm{x})=\sum_{j=1}^{\mathrm{k}} \frac{\alpha_{j}^{\mathrm{s}(\mathrm{j})}}{\left(\mathrm{x}-\mathrm{a}_{\mathrm{j}}\right)} ; \text { and }
$$

(3) $\theta=\omega+\left(\mathrm{P}^{\prime} / \mathrm{P}\right)$.

The above algorithm will generate $t w$, uistinct $\theta$ 's iff the three groups in (a) above are simultaneously diagonalizable. If there are no such solutions these groups are irreducible. 
Proof: The statement in (a) is just a combination of Proposition 2.2(e) and the first part of Theorem 3.5, and similarly for simultaneous diagonalizability of these groups. For the equivalence of (a) and (b) see [10, pp. 7-8]. The algorithm given in steps (1)-(3) for finding solutions of the form $\mathrm{y}=\exp \left(\int \theta\right)$ with $\theta \in \mathbb{C}(\mathrm{x})$ is a restatement of $[10$, pp. 11-12] for the Fuchsian case. The remainder of the theorem can be found in the proof in Kovacic [10, pp. 15-17], with the uniqueness of $P$ in (2) being shown by calculating the Wronskian of the two solutions.

Q.E.D.

Such reducibility criteria have a long history; for example see [15, pp. 176-178] which was published in 1897. For the Fuchsian case with three regular singular points (e.g., the hypergeometric equation), simpler formulations are available (see Theorem 2.24 of [2]). It should be noted that Kovacic's algorithm is not restricted to the Fuchsian case of (4.2).

4.10 Corollary: Assume the reducibility algorithm implicit in steps (1)-(3) of Theorem 4.9 has yielded (1) only one solution, (2) two independent solutions of (4.2) with the form $y=\exp \left(\int \theta\right)$ where $\theta=\sum_{j=1}^{k} \frac{\alpha_{j}^{s(j)}}{\left(x-a_{j}\right)}+\left(P^{\prime} / P\right)$.

Then:

(a) In case (1) the monodromy group $G_{M}$ of (4.1) is a Ziglin group iff each of the numbers $\left[\bar{A}_{j}+2 \alpha_{j}^{s(j)}\right], j=1,2, \ldots, k$, is rational.

(b) In case (2) $G_{M}$ is a Ziglin group iff there are integers $m$ and $n$ so that $\left[(m+n) A_{j}+2(m-n) \alpha_{j}^{s(j)}\right]$ is an even integer for $j=1,2, \ldots, k$. In particular, $G_{M}$ is Ziglin if each of the numbers $\left[A_{j} \pm 2 \alpha_{j}^{s(j)}\right], j=1,2, \ldots$, $\mathrm{k}$, is rational. 
Proof: In terms of a basis $\{*, y\}$ of germs of solutions of (4.2) at $\mathrm{x}_{0}$

we have

$$
N_{j}=\left[\begin{array}{cc}
\exp \left(2 \pi i \alpha_{j}^{s(j)}\right) & 0 \\
* & \exp \left(-2 \pi i \alpha_{j}^{s(j)}\right)
\end{array}\right] .
$$

The result then follows from (4.7) and Theorem 3.5 (a) and (b) respectively.

Q.E.D.

Case II (The DP-Case):

The algorithm is stated in terms of the following sets:

$$
\left\{\begin{array}{l}
\mathrm{E}_{\mathrm{j}}=\left\{2+\mathrm{e}\left(1+4 \beta_{\mathrm{j}}\right)^{\frac{1}{2}} \mid \mathrm{e}=0, \pm 2\right\} \cap \mathbb{Z} \text { if } \beta_{\mathrm{j}} \neq 0, \\
\mathrm{E}_{\mathrm{j}}=\{4\} \quad \text { if } \beta_{\mathrm{j}}=0, \delta_{\mathrm{j}} \neq 0, \\
\mathrm{E}_{\mathrm{j}}=\{0\} \quad \text { if } \beta_{\mathrm{j}}=0=\delta_{\mathrm{j}}
\end{array}\right.
$$

and

$$
\left\{\begin{array}{l}
\mathrm{E}_{\infty}=\left\{2+\mathrm{e}\left(1+4 \beta_{\infty}\right)^{\frac{1}{2}} \mid \mathrm{e}=0, \pm 2\right\} \cap \mathbb{Z} \text { if } \beta_{\infty} \neq 0 \\
\mathrm{E}_{\infty}=\{0,2,4\} \text { if } \beta_{\infty}=0 .
\end{array}\right.
$$

4.13 Theorem: The following two statements are equivalent:

(a) $G_{M}, G_{N}$, and $G_{D}$ are irreducible (i.e., Case I does not hold) but are simultaneously DP-groups.

(b) There is a solution of (4.2) of the form $y=\exp \left(\int \omega\right)$, where $\omega$ is algebraic over $\mathbb{C}(\mathrm{x})$ of degree 2 , and Case I does not hold.

Moreover, there is a solution as in (b) iff

(1) there is a choice of $\mathrm{e}_{\mathrm{j}} \varepsilon \mathrm{E}_{\mathrm{j}}$ and $\mathrm{e}_{\boldsymbol{w}} \varepsilon \mathrm{E}_{\boldsymbol{w}}$ which are not all even integers so that $d=(1 / 2)\left[e_{\infty}-\sum_{j=1}^{k} e_{j}\right]$ is a non-negative integer; and 
(2) there is a monic, degree $d$ polynomial $P$ (which can be found by the method of undetermined coefficients) satisfying

$$
\begin{aligned}
& \mathrm{P}^{\prime \prime \prime}+3 \theta \mathrm{P}^{\prime \prime}+\left(3 \theta^{2}+3 \theta^{\prime}-4 \mathrm{r}\right) \mathrm{P}^{\prime}+\left(\theta^{\prime}+3 \theta \theta^{\prime}+\theta^{3}-4 \mathrm{r} \theta-2 \mathrm{r}^{\prime}\right) \mathrm{P}=0 \\
& \text { where } \theta=(1 / 2) \sum_{\mathrm{j}=1}^{\mathrm{k}} \frac{\mathrm{e}_{\mathrm{j}}}{\left(\mathrm{x}-\mathrm{a}_{\mathrm{j}}\right)}
\end{aligned}
$$

Specifically, for $\mathrm{P}$ as in (2) let $\phi=\theta+\left(\mathrm{P}^{\prime} / \mathrm{P}\right)$ and choose a solution $\omega$ of $\omega^{2}+\phi \omega+\left[(1 / 2) \phi^{\prime}+(1 / 2) \phi^{2}-\mathrm{r}\right]=0$; then $\mathrm{y}=\exp \left(\int \omega\right)$ will be a solution of $(4.2)$ as in (b) above.

Proof: For the equivalence of (a) and (b) see [10, pp. 7-8]. The remainder is a restatement of $[10$, p. 18] for the Fuchsian case.

Q.E.D.

4.14 Corollary: Assume $G_{D}$ is irreducible, but that the DP-algorithm implicit in (1)-(2) and the final statement of Theorem 4.13 results in a solution of the required form. Then the monodromy group $G_{M}$ of (4.1) is Ziglin iff all $A_{j}$ are rational.

Proof: This follows from (4.7) and Theorem 3.5(c).

Q.E.D.

4.15 Remark: A necessary condition for the algorithm of Theorem 4.13 to give a solution of the required form is that in (4.4) some $\beta_{\mathrm{j}} \neq 0$ (see $[10$, p. 8]). Thus, if all $\beta_{\mathrm{j}}=0$ one need examine only Cases I and III.

\section{Case III (The Finite Case):}

The algorithm is stated in terms of the following sets, where $\mathrm{n}=4$, 6 , or 12 : 


$$
\left\{\begin{array}{l}
F_{j}(n)=\left\{6+(12 e / n)\left(1+4 \beta_{j}\right)^{\frac{1}{2}} \mid e=0, \pm 1, \ldots, \pm(n / 2)\right\} \cap \mathbb{I} \text { if } \beta_{j} \neq 0, \\
F_{j}(n)=\{12\} \text { if } \beta_{j}=0, \delta_{j} \neq 0, \\
F_{j}(n)=\{0\} \text { if } \beta_{j}=0=\delta_{j},
\end{array}\right.
$$

and

$$
\begin{aligned}
& \mathrm{F}_{\infty}(\mathrm{n})=\left\{6+(12 \mathrm{e} / \mathrm{n})\left(1+4 \beta_{\infty}\right)^{\frac{1}{2}} \mid \mathrm{e}=0, \pm 1, \ldots, \pm(\mathrm{n} / 2)\right\} \cap \mathbb{Z}, \\
& \text { regardless of whether or not } \beta_{\infty}=0 .
\end{aligned}
$$

4.18 Theorem: Assume $G_{D}$ is not reducible and not a DP-group (i.e., Cases I and II do not hold). Then the following procedure will determine if $G_{D}$ is finite with all solutions of (4.2) being algebraic over $\mathbb{C}(\mathrm{x})$.

(1) Let $n=4$ and write down all choices of $f_{j} \in F_{j}(n)$ and $f_{\infty} \varepsilon F_{\infty}(n)$ for which $d=(n / 12)\left[f_{\infty}-\sum_{j=1}^{k} f_{j}\right]$ is a non-negative integer;

(2) for each such choice set $\theta=(n / 12) \sum_{j=1}^{k} \frac{f_{j}}{\left(x-a_{j}\right)}$, and with $S=\Pi\left(x-a_{j}\right)$ (where the product is taken over only those $a_{j}$ which are poles of $r(x)$ ) determine (e.g., by the method of undetermined coefficients) if there is a monic, degree $d$ polynomial $P$ such that if we set $P_{n}=-P$ and recursively define

$$
\mathrm{P}_{\mathrm{i}-1}=-\mathrm{SP}_{\mathrm{i}}^{\prime}+\left[(\mathrm{n}-\mathrm{i}) \mathrm{S}^{\prime}-\mathrm{S} \theta\right] \mathrm{P}_{\mathrm{i}}-(\mathrm{n}-\mathrm{i})(\mathrm{i}+1) \mathrm{S}^{2} \mathrm{r} \mathrm{P}_{\mathrm{i}+1}
$$

for $\mathrm{i}=\mathrm{n}, \mathrm{n}-1, \ldots, 0$, then $\mathrm{P}_{-1} \equiv 0$;

(3) repeat, if necessary, steps (1) and (2) with $\mathrm{n}=6$ and then with $\mathrm{n}=12$; and 
(4) if such a $P$ is found in (2) for $n=4,6$, or 12 , then $G_{D}$ is finite. Moreover, a solution $\omega$ to the equation $\sum_{i=0}^{n} \frac{S^{i} P_{i}}{(n-i) !} \omega^{i}=0$ will give a solution $\mathrm{y}=\exp \left(\int \omega\right)$ to $(4.2)$.

Proof: See [10, pp. 7-8 and pp. 22-23] and recall that (4.2) is Fuchsian.

Q.E.D.

4.19 Corollary: Assume $G_{D}$ is irreducible and not a DP-group, but is finite. Then $G_{M}$ is Ziglin.

Proof: $\quad$ By Theorem 3.5(d).

Q.E.D.

4.20 Remarks: A success in the algorithm (1)-(4) of Theorem 4.19 implies $G_{D}$ is finite and projectively the tetrahedral $(\mathrm{n}=4)$, octahedral $(\mathrm{n}=6)$, or iscosahedral $(n=12)$ group (see $\left[10\right.$, p. 27]). A failure implies that $G_{D}=S L(2, \mathbb{C})$

(see $[10$, p. 7$]$ ). Necessary conditions for a success in the algorithm of Theorem 4.19 are that all the characteristic exponents $\tau_{\mathrm{j}}^{ \pm}$and $\tau_{\infty}^{ \pm}$of (4.6) be rational; that is, all $\left(1+4 \beta_{\mathrm{j}}\right)^{\frac{1}{2}}$ and $\left(1+4 \beta_{\infty}\right)^{\frac{1}{2}}$ are rational (see $[10$, p. 8$]$ ). If this does not hold, only Cases I and II need to be examined.

\section{Applications}

(A) Let $\mathrm{a}(\mathrm{x}), \mathrm{b}(\mathrm{x})$, and $\mathrm{c}(\mathrm{x}, \mathrm{y})$ be arbitrary meromorphic functions on $\mathbb{C}^{2}=$ $\{(\mathrm{x}, \mathrm{y})\}$, let $\mathrm{h} \in \mathbb{C}$, and consider the analytic set.

$$
\gamma=\left\{(\mathrm{x}, \mathrm{y}) \varepsilon \mathbb{C}^{2} \mid \mathrm{b}(\mathrm{x}) \mathrm{c}(\mathrm{x}, \mathrm{y})=\mathrm{h}\right\}
$$

As an example, if $\mathrm{b}(\mathrm{x})$ is a separable polynomial of positive degree $(2 \mathrm{~g}+1)$ or $(2 \mathrm{~g}+2), \quad \mathrm{h}=1$, and $\mathrm{c}(\mathrm{x}, \mathrm{y})=\mathrm{y}^{-2}$, then $\gamma$ is a punctured algebraic curve of genus $\mathrm{g}$. Returning to generalities, suppose $\Gamma c \gamma$ is a Riemann surface on which $a(x) / b(x)$ is 
finite, and having the property that the projection $\pi(\mathrm{x}, \mathrm{y})=\mathrm{x}$ of $\Gamma$ into $\mathbb{C}$ is unbounded. Via the embedding $(x, y) \rightarrow(x, 0, y, 0)$ we may view

$\mathrm{E}=\left\{\left(\mathrm{x}, \xi_{2}, \mathrm{y}, \eta_{2}\right) \mid(\mathrm{x}, \mathrm{y}) \in \Gamma\right\} \subset \mathbb{C}^{4}$ with projection $\left(\mathrm{x}, \xi_{2}, \mathrm{y}, \eta_{2}\right) \rightarrow(\mathrm{x}, \mathrm{y})$ as a rank two complex vector bundle over $\Gamma$. A holomorphic connection $\nabla$ can then be defined on E through the local coordinate representation

$$
\mathrm{d}\left[\begin{array}{l}
\xi_{2} \\
\eta_{2}
\end{array}\right]=\left[\begin{array}{cc}
0 & 1 \\
-\mathrm{a}(\mathrm{x}) & 0 \\
\mathrm{~b}(\mathrm{x}) & 0
\end{array}\right]\left[\begin{array}{l}
\xi_{2} \\
\eta_{2}
\end{array}\right] \mathrm{dx} .
$$

$\nabla$ is designed so as to be the pullback of

$$
\xi^{\prime \prime}+\frac{\mathrm{a}(\mathrm{x})}{\mathrm{b}(\mathrm{x})} \xi=0, \quad \xi=\xi_{2}, \quad,=(\mathrm{d} / \mathrm{dx})
$$

under the projection $\pi: \Gamma \rightarrow \mathbb{C}$ (see $[2$, Section 4]). As a consequence, the monodromy group $M$ of $\nabla$ embeds into the monodromy group $G_{N}$ of (5.3) (see $[4$, Proposition 1.1]). If $\mathrm{G}_{\mathrm{N}}$ is Ziglin, then Proposition 3.1(b) implies that $\mathrm{M}$ is Ziglin.

There are simple instances in which one can view the projection $\pi: \Gamma \rightarrow \mathbb{C}$ as a reduction w.r.t. a finite symmetry group G. For example, if $\Gamma$ is invariant under each mapping

$$
(\exp (2 \pi \mathrm{ij} / \mathrm{n}),(\mathrm{x}, \mathrm{y})) \rightarrow(\mathrm{x}, \exp (2 \pi \mathrm{ij} / \mathrm{n}) \cdot \mathrm{y}), \quad \mathrm{j}=1,2, \ldots, \mathrm{n}
$$

as would be the case if $\mathrm{c}(\mathrm{x}, \mathrm{y})=\mathrm{y}^{ \pm \mathrm{n}}$, then Theorem 1.2 can be applied (the group action on the fibers of $\mathrm{E} \rightarrow \Gamma$ being the identity map in the $\left(\xi_{2}, \eta_{2}\right)$-coordinates of $(5.2))$.

The algorithm of Section 4 applies directly to (5.3) when $r(x)=-[a(x) / b(x)]$ is of the form given in (4.4). For exarripie, suppose

$$
r(x)=\left[\frac{1}{x^{2}}+\frac{1}{(x-1)^{2}}+\frac{1}{(x-2)^{2}}-\frac{1}{x}+\frac{1}{(x-1)}\right] .
$$


Then: (I) there is no choice of the $\alpha_{j}^{ \pm}=(1 / 2)[1 \pm \sqrt{5}]$ and $\alpha_{\infty}^{ \pm}=(1 / 2)[1 \pm \sqrt{17}]$ which makes the $\mathrm{d}$ of Theorem 4.9(1) a non-negative integer; (II) all $\mathrm{E}_{\mathrm{j}}=\{2\}=\mathrm{E}_{\infty}$ so that there are no choices of the $e_{j}$ and $e_{\infty}$, not all even, with which to construct a non-negative $\mathrm{d}$ as in Theorem 4.13(1); and (III) $\beta_{1}=1$ implies $\left(1+4 \beta_{1}\right)^{\frac{1}{2}}=\sqrt{5}$ is not rational so that Case III does not apply (see the necessary conditions in Remark 4.20). The differential Galois group $G_{D}$ of $(5.3)-(5.4)$ is then $\operatorname{SL}(2, \mathbb{C})$. Recalling Proposition 2.2(d), we see that by Proposition 3.1(d) and Corollary 3.4 the monodromy group $\mathrm{G}_{\mathrm{M}}=\mathrm{G}_{\mathrm{N}}$ of $(5.3)-(5.4)$ cannot be Ziglin. If (5.3)-(5.4) is achieved from the $\nabla$ of (5.2) by reduction using a finite group (see the previous paragraph for an example), then by Theorem 1.2 the monodromy group $M$ of $\nabla$ is also not Ziglin.

To fit the above into a Hamiltonian context, give $\mathbb{C}^{4}=\left\{\left(\mathrm{x}, \mathrm{x}_{2}, \mathrm{y}, \mathrm{y}_{2}\right)\right\}$ the standard symplectic structure $\omega=\mathrm{dx} \wedge \mathrm{dy}+\mathrm{dx}_{2} \wedge \mathrm{dy}_{2}$, let $\mathrm{p}(\mathrm{x}, \mathrm{y})=\mathrm{a}(\mathrm{x})(\partial \mathrm{c} / \partial \mathrm{y})(\mathrm{x}, \mathrm{y})$ and $q(x, y)=b(x) c(x, y)$, and consider the Hamiltonian

$$
\mathrm{H}\left(\mathrm{x}, \mathrm{x}_{2}, \mathrm{y}, \mathrm{y}_{2}\right)=\mathrm{q}(\mathrm{x}, \mathrm{y})+(1 / 2) \mathrm{p}(\mathrm{x}, \mathrm{y}) \mathrm{x}_{2}^{2}+(1 / 2)(\partial \mathrm{q} / \partial \mathrm{y})(\mathrm{x}, \mathrm{y}) \mathrm{y}_{2}^{2}+\mathrm{o}_{3}\left(\mathrm{x}_{2}, \mathrm{y}_{2}\right) .
$$

The associated vector field $X_{H}$ is tangent to the $(x, y)$-plane, a phase curve $\Gamma$ in that plane of energy $h$ is contained in the set $\gamma$ of (5.1), and the normal variational equation (NVE) along $\Gamma^{-}$may be identified with $(5.2)$ where $\left(\xi_{2}, \eta_{2}\right)$ are the (global) linearized variables associated to $\left(\mathrm{x}_{2}, \mathrm{y}_{2}\right)$ (see [2, Section 4]). In the context of the previous paragraphs, conclusions about the non-integrability of $X_{H}$ can be drawn from Corollary 1.3.

(B) For our second example we illustrate how reduction can be combined with Kovacic's algorithm to explicitly compute the monodromy of a holomorphic connection that arises in a Hamiltonian system.

First consider the Fuchsian equation 


$$
y^{\prime \prime}+p(x) y^{\prime}+q(x) y=0, \quad, \quad=(d / d x)
$$

on $\mathbb{C}$ with

$$
\left\{\begin{array}{l}
p(x)=\left[\frac{1}{x}+\frac{(1 / 2)}{(x-1)}\right] \\
q(x)=\left[-\frac{1}{x^{2}}+\frac{(1 / 2)}{x}-\frac{(1 / 2)}{(x-1)}\right]
\end{array}\right.
$$

and the associated normal form

$$
y^{\prime \prime}=r(x) y \quad, \quad r(x)=\left[\frac{(3 / 4)}{x^{2}}-\frac{(3 / 16)}{(x-1)^{2}}+\frac{(3 / 4)}{x(x-1)}\right]
$$

Applying Case I of Section 4 we find only two choices of the $\alpha^{ \pm}$for which the $d$ in Theorem $4.9(1)$ is a non-negative integer:

$$
\begin{gathered}
\left\{\alpha_{\infty}^{+}, \alpha_{1}^{+}, \alpha_{2}^{+}\right\}=\{7 / 4,3 / 2,1 / 4\} \text { with } d=0, \\
\left\{\alpha_{\infty}^{+}, \alpha_{1}^{-}, \alpha_{2}^{-}\right\}=\{7 / 4,-1 / 2,1 / 4\} \text { with } d=2 .
\end{gathered}
$$

Both cases lead to the single solution

$$
y(x)=x^{3 / 2}(x-1)^{\frac{1}{4}}
$$

of (5.8).

From the proof of Corollary 4.10 we see that the monodromy group $G_{N}$ of (5.8) is generated by $N_{1}=\left[\begin{array}{rr}-1 & 0 \\ -a & -1\end{array}\right]$ and $N_{2}=\left[\begin{array}{rr}i & 0 \\ b & -i\end{array}\right]$. Note that $a \neq 0$ in $N_{1}$ since otherwise the distinct eigenvalues of $\mathrm{N}_{2}$ would imply that $\mathrm{G}_{\mathrm{N}}$ was diagonalizable, contrary to the algorithm giving us only the one solution (5.9) in Case I. Now recall from (4.7) that the monodromy group $G_{M}$ of (5.6) is generated 
by $\mathrm{M}_{\mathrm{j}}=\theta_{\mathrm{j}} \mathrm{N}_{\mathrm{j}}$ where $\theta_{\mathrm{j}}=\exp \left(\pi \mathrm{i} \mathrm{A}_{\mathrm{j}}\right), \mathrm{j}=1,2$, with the $\mathrm{A}_{\mathrm{j}}$ defined as in (4.3). From $p(x)$ in (5.7) we see that $A_{1}=1$ and $A_{2}=1 / 2$, and so $M_{1}=-N_{1}=\left[\begin{array}{ll}1 & 0 \\ a & 1\end{array}\right]$ and $\mathrm{M}_{2}=\mathrm{iN}_{2}=\left[\begin{array}{cc}-1 & 0 \\ \mathrm{ib} & 1\end{array}\right]$.

Equation (5.6) with (5.7) occurs in a Hamiltonian context somewhat as in Example (A). Using the standard symplectic structure on $\mathbb{C}^{4}$ and

$$
\mathrm{H}\left(\mathrm{x}_{1}, \mathrm{x}_{2}, \mathrm{y}_{1}, \mathrm{y}_{2}\right)=(1 / 2)\left(\mathrm{y}_{1}^{2}+\mathrm{y}_{2}^{2}\right)+(1 / 2)\left(\mathrm{x}_{1}^{2}+\mathrm{x}_{2}^{2}\right)+(2 / 3) \mathrm{x}_{1}^{3}+\mathrm{x}_{1} \mathrm{x}_{2}^{2}
$$

the associated vector field $\mathrm{X}_{\mathrm{H}}$ is tangent to the $\left(\mathrm{x}_{1}, \mathrm{y}_{1}\right)$-plane, and there is a phase curve $\Gamma$ within this plane at energy $h=0$ contained in the algebraic curve defined by

$$
y_{1}^{2}=x_{1}^{2}\left[-(4 / 3) x_{1}-1\right]
$$

The $\mathbb{Z}_{2}$ - action $\left(\mathrm{x}_{1}, \mathrm{y}_{1}\right) \rightarrow\left(\mathrm{x}_{1},-\mathrm{y}_{1}\right)$ preserves $(5.11)$ and lifts to a symmetry of the NVE $\nabla$ along $\Gamma$, where $\nabla$ is the pullback of (5.6)-(5.7) under the reduction mapping $\left(x_{1}, y_{1}\right) \rightarrow-(4 / 3) x_{1}=x$. (Analogous computations are done in Example $C$ of [4], pp. 110-112.) This mapping is unbranched over $\mathrm{x}=0$ and branched with order 2 over $\mathrm{x}=1$. Since $\mathrm{a} \neq 0$ and $\mathrm{M}_{2}^{2}=\mathrm{id}$, we see that the monodromy group of $\nabla$ is isomorphic to the infinite-cyclic group generated by $M_{1}$.

The Hamiltonian (5.10) is but one member of a family that has been extensively studied using Ziglin analysis (see [8, Theorem 4, p. 472] and [13, Corollary 1, p. 266]), which in this case fails to detect the non-existence of a second independent integral. One might have anticipated some degeneracy in the monodromy of $\nabla$ from the fact that the above Hamiltonian (5.10) and one which is known to be completely integrable have the same linearized equations about solutions in the $\left(\mathrm{x}_{1}, \mathrm{y}_{1}\right)$-plane (see [12, Section 5]). However, the integrability status of (5.10) is unknown. For another example of a Hamiltonian system which is completely integrable with infinite cyclic monodromy group for its NVE, see [3, Section 5]. For 
other examples in Ziglin analysis we refer to $[3,4]$ and the surveys $[14,19]$.

\section{$\underline{\text { References }}$}

1. D.G. Babbitt and V.S. Varadarajan, "Local Moduli for Meromorphic Differential Equations", Astérisque 169-170 (1989).

2. A. Baider and R.C. Churchill, "On Monodromy Groups of Second Order Fuchsian Equations", to appear in SIAM J. of Math. Analysis.

3. A. Baider, R.C. Churchill, and D.L. Rod, "Monodromy and Non-Integrability in Complex Hamiltonian Systems", to appear in J. of Dynamics and Differential Equations.

4. R.C. Churchill and D.L. Rod, "Geometrical Aspects of Ziglin's Non-Integrabiiity Theorem for Complex Hamiltonian Systems", J. of Differential Equations 76(1988), 91-114.

5. A. Duval and M. Loday-Richaud, "A propos de l'algorithme de Kovacic", preprint 89-12, Université de Paris-Sud, 1989.

6. W. Fulton, "Algebraic Curves", Benjamin/Cummings, Reading, MA, 1969.

7. J. Gray, "Linear Differential Equations and Group Theory from Riemann to Poincare", Birkhäuser, Boston, MA, 1986.

8. H. Ito, "A Criterion for Non-Integrability of Hamiltonian Systems with Non-homogeneous Potentials", J. of Applied Math. and Physics (ZAMP) 38(1987), 459-476.

9. I. Kaplansky, "An Introduction to Differential Algebra", secoi: edition, Hermann, Paris, 1976.

10. J. Kovacic, "An Algorithm for Solving Second Order Linear Homogeneous Differential Equations", J. Symbolic Computation 2(1986), 3-43. 
11. L. Markus, "Group Theory and Differential Equations", Lecture Notes at the University of Minnesota, 1959-1960.

12. A. Ramani, B. Grammaticos, and H. Yoshida, "Rigorous Non-Integrability Results Related to Singularity Analysis", in "Proceedings of Conference at Como, 1988" (A.P. Fordy, Ed.) Manchester University Press, 1990.

13. D.L. Rod, "On a Theorem of Ziglin in Hamiltonian Dynamics", in "Hamiltonian Dynamical Systems" (Kenneth R. Meyer and Donald E. Saari, Eds.), 259-270, Contemporary Mathematics, Vol.81, American Mathematical Society, Providence, R.I., 1988.

14. D.L. Rod and R.C. Churchill, "On the Applicability. of Ziglin's Non-Integrability Theorem", in "Proceedings of the Workshop on Finite Dimensional Integrable Nonlinear Dynamical Systems" (P.G.L. Leach and W H. Steeb, Eds.), 94-109, World Scientific, Singapore, 1988.

15. L. Schlesinger, "Handbuch der Theorie der linearen Differentialgleichungen", Leipzig, 1897.

16. M. Singer, "An Outline of Differential Galois Theory", in "Computer Algebra and Differential Equations" (E.Tournier, Ed.), Academic Press, 1990.

17. M. Singer, "Formal Solutions of Differential Equations", preprint, Version III, August 11, 1989.

18. C. Tretkoff and M. Tretkoff, "Solution of the Inverse Problem of Differential Galois Theory in the Classical Case", American J. of Math. 101(1979), 1327-1332.

19. H. Yoshida, "Ziglin Analysis for Proving :uir-integrability of Hamiltonian Systems", in "Proceedings of the Workshop on Finite Dimensional Integrable Nonlinear Dynamical Systems" (P.G.L. Leach and W.H. Steeb, Eds.), 74-93, World Scientific, Singapore, 1988. 
20. S.L. Ziglin, "Branching of Solutions and Non-existence of First Integrals in Hamiltonian Mechanics I and II", Functional Anal. Appl. 16(1982), 181-189, and 17(1983), 6-17.

21. Morales Ruiz, Juan Jose, "Tecnicas Algebraicas para el Estudio de la Integrabilidad de Sistemas Hamiltonianos", Ph. D. Thesis, University of Barcelona, December 16, 1989. 
Li Ta-Tsien (Li Da-qian) and Zhao Yan-Chun, Global Existence of Classical Solutions to the Typical Free Boundary Problem for General Quasilinear Hyperbolic Systems and its Applications

Thierry Cazenave and Fred B. Weissler, The Structure of Solutions to the Pseudo-Conformally Invariant Nonlinear Schrödinger Equation

Marshall Slemrod and Athanasios E. Tzavaras, A Limiting Viscosity Approach for the Riemann Problem in Isentropic Gas Dynamics

Richard D. James and Scott J. Spector, The Formation of Filamentary Voids in Solids

P.J. Vassiliou, On the Geometry of Semi-Linear Hyperbolic Partial Differential Equations in the Plane Integrable by the Method of Darboux

Jerome V. Moloney and Alan C. Newell, Nonlinear Optics

Keti Tenenblat, A Note on Solutions for the Intrinsic Generalized Wave and Sine-Gordon Equations

P. Szmolyan, Heteroclinic Orbits in Singularly Perturbed Differential Equations

Wenxiong Liu, A Parabolic System Arising In Film Development

Daniel B. Dix, Temporal Asymptotic Behavior of Solutions of the Benjamin-Ono-Burgers Equation

Michael Renardy and Yuriko Renardy, On the nature of boundary conditions for flows with moving free surfaces

Werner A. Stahel, Robust Statistics: From an Intellectual Game to a Consumer Product

Avner Friedman and Fernando Reitich, The Stefan Problem with Small Surface Tension

E.G. Kalnins and W. Miller, Jr., Separation of Variables Methods for Systems of Differential Equations in Mathematical Physics

Mitchell Luskin and George R. Sell, The Construction of Inertial Manifolds for Reaction-Diffusion Equations by Elliptic Regularization

Konstantin Mischaikow, Dynamic Phase Transitions: A Connection Matrix Approach

Philippe Le Floch and Li Tatsien, A Global Asymptotic Expansion for the Solution to the Generalized Riemann Problem

Matthew Witten, Ph.D., Computational Biology: An Overview

Matthew Witten, Ph.D., Peering Inside Living Systems: Physiology in a Supercomputer

Michael Renardy, An existence theorem for model equations resulting from kinetic theories of polymer solutions

Daniel D. Joseph and Luigi Preziosi, Reviews of Modern Physics: Addendum to the Paper "Heat Waves"

Luigi Preziosi, An Invariance Property for the Propagation of Heat and Shear Waves Time Effects

Prabir Daripa, On the Computation of the Beltrami Equation in the Complex Plane

Philippe Le Floch, Shock Waves for Nonlinear Hyperbolic Systems in Nonconservative Form

A.L. Gorin, D.B. Roe and A.G. Greenberg, On the Complexity of Pattern Recognition Algorithms On a Tree-Structured Parallel Computer

Mark J. Friedman and Eusebius J. Doedel, Numerical computation and continuation of invariant manifolds connecting fixed points

Scott J. Spector, Linear Deformations as Global Minimizers in Nonlinear Elasticity

Denis Serre, Richness and the classification of quasilinear hyperbolic systems

L. Preziosi and F. Rosso, On the stability of the shearing flow between pipes

Avner Friedman and Wenxiong Liu, A system of partial differential equations arising in electrophotography

Jonathan Bell, Avner Friedman, and Andrew A. Lacey, On solutions to a quasilinear diffusion problem from the study of soft tissue

David G. Schaeffer and Michael Shearer, Loss of hyperbolicity in yield vertex plasticity models under nonproportional loading

Herbert C. Kranzer and Barbara Lee Keyfitz, A strictly hyperbolic system of conservation laws admitting singular shocks

S. Laederich and M. Levi, Qualitative dynamics of planar chains

Milan Miklavčič , A sharp condition for existence of an inertial manifold

Charles Collins, David Kinderlehrer, and Mitchell Luskin, Numerical approximation of the solution of a variational problem with a double well potential

Todd Arbogast, Two-phase incompressible flow in a porous medium with various nonhomogeneous boundary conditions

Peter Poláčik, Complicated dynamics in scalar semilinear parabolic equations in higher space dimension 
Bei Hu, Diffusion of penetrant in a polymer: a free boundary problem

Mohamed Sami ElBialy, On the smoothness of the linearization of vector fields near resonant hyperbolic rest points

Max Jodeit, Jr. and Peter J. Olver, On the equation $\operatorname{grad} f=M \operatorname{grad} g$

Shui-Nee Chow, Kening Lu, and Yun-Qiu Shen, Normal form and linearization for quasiperiodic systems

Prabir Daripa, Theory of one dimensional adaptive grid generation

Michael C. Mackey and John G. Milton, Feedback, delays and the origin of blood cell dynamics

D.G. Aronson and S. Kamin, Disappearance of phase in the Stefan problem: one space dimension

Martin Krupa, Bifurcations of relative equilibria

D.D. Joseph, P. Singh, and K. Chen, Couette flows, rollers, emulsions, tall Taylor cells, phase separation and inversion, and a chaotic bubble in Taylor-Couette flow of two immiscible liquids

Artemio González-López, Niky Kamran, and Peter J. Olver, Lie algebras of differential operators in two complex variables

L.E. Fraenkel, On a linear, partly hyperbolic model of viscoelastic flow past a plate

Stephen Schecter and Michael Shearer, Undercompressive shocks for nonstrictly hyperbolic conservation laws

Xinfu Chen, Axially symmetric jets of compressible fluid

J. David Logan, Wave propagation in a qualitative model of combustion under equilibrium conditions

M.L. Zeeman, Hopf bifurcations in competitive three-dimensional Lotka-Volterra Systems

Allan P. Fordy, Isospectral flows: their Hamiltonian structures, Miura maps and master symmetries

Daniel D. Joseph, John Nelson, Michael Renardy, and Yuriko Renardy, Two-Dimensional cusped interfaces

Avner Friedman and Bei Hu, A free boundary problem arising in electrophotography

Hamid Bellout, Avner Friedman and Victor Isakov, Stability for an inverse problem in potential theory

Barbara Lee Keyfitz, Shocks near the sonic line: A comparison between steady and unsteady models for change of type

Barbara Lee Keyfitz and Gerald G. Warnecke, The existence of viscous profiles and admissibility for transonic shocks

P. Szmolyan, Transversal heteroclinic and homoclinic orbits in singular perturbation problems

Philip Boyland, Rotation sets and monotone periodic orbits for annulus homeomorphisms

Kenneth R. Meyer, Apollonius coordinates, the N-body problem and continuation of periodic solutions

Chjan C. Lim, On the Poincare-Whitney circuitspace and other properties of an incidence matrix for binary trees

K.L. Cooke and I. Györi, Numerical approximation of the solutions of delay differential equations on an infinite interval using piecewise constant arguments

Stanley Minkowitz and Matthew Witten, Periodicity in cell proliferation using an asynchronous cell population

M. Chipot and G. Dal Maso, Relaxed shape optimization: The case of nonnegative data for the Dirichlet problem

Jeffery M. Franke and Harlan W. Stech, Extensions of an algorithm for the analysis of nongeneric Hopf bifurcations, with applications to delay-difference equations

Xinfu Chen, Generation and propagation of the interface for reaction-diffusion equations

Philip Korman, Dynamics of the Lotka-Volterra systems with diffusion

Harlan W. Stech, Generic Hopf bifurcation in a class of integro-differential equations

Stephane Laederich, Periodic solutions of non linear differential difference equations

Peter J. Olver, Canonical Forms and Integrability of BiHamiltonian Systems

S.A. van Gils, M.P. Krupa and W.F. Langford, Hopf bifurcation with nonsemisimple 1:1 Resonance

R.D. James and D. Kinderlehrer, Frustration in ferromagnetic materials

Carlos Rocha, Properties of the attractor of a scalar parabolic P.D.E.

Debra Lewis, Lagrangian block diagonalization

Richard C. Churchill and David L. Rod, On the determination of Ziglin monodromy groups 